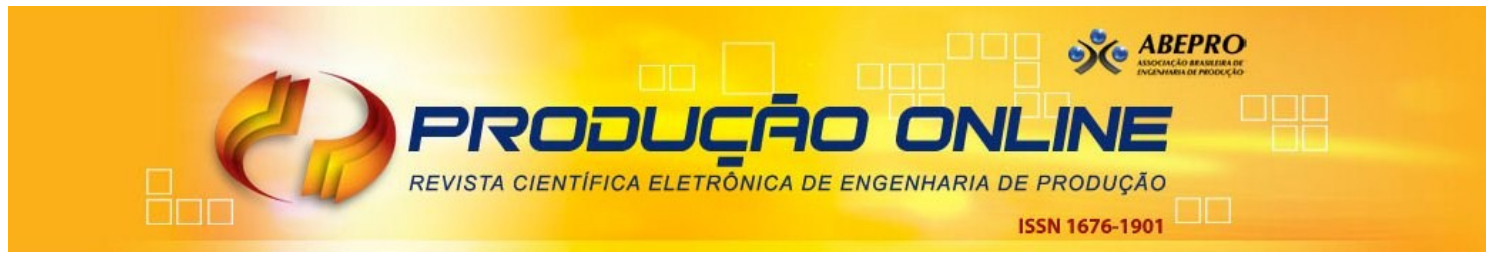

\title{
CINTOS LOMBARES NO AMBIENTE OCUPACIONAL: QUAIS SÃO AS EVIDÊNCIAS?
}

\section{BACK BELTS IN OCCUPATIONAL SETTING: WHAT ARE THE EVIDENCES?}

\author{
João Marcos Bernardes * E-mail: jmbernardes@yahoo.com \\ Antônio Renato Pereira Moro *E-mail: moro@cds.ufsc.br \\ *Universidade Federal de Santa Catarina, UFSC, Florianópolis, SC
}

\begin{abstract}
Resumo: Embora largamente utilizados, a eficácia dos cintos lombares na prevenção de distúrbios da coluna lombar permanece incerta, assim como seus mecanismos de ação, que não foram ainda completamente comprovados, suscitando controvérsia quanto a sua utilização. Deste modo, este estudo teve como objetivo realizar uma revisão crítica da literatura sobre o uso de cintos lombares na prevenção da dor lombar no ambiente ocupacional. Para tanto foram realizadas pesquisas nas seguintes bases de dados: Medline, SciELO e LILACS. Existem evidências que o uso dos cintos lombares reduz as amplitudes de movimento da coluna, entretanto os estudos referentes à pressão intra-abdominal e à atividade muscular apresentam resultados contraditórios, assim como os estudos epidemiológicos, não permitindo, desta forma a recomendação do seu uso na prevenção da dor lombar de origem ocupacional. Com base nesta revisão de literatura sugere-se, portanto a realização de novos estudos, a fim de confirmar ou não a eficácia dos cintos lombares como equipamento de proteção individual na prevenção dos distúrbios lombares de origem ocupacional.
\end{abstract}

Palavras-chave: Ergonomia, saúde ocupacional, riscos ocupacionais, equipamentos de proteção individual, dor lombar.

Abstract: Although, widely used the mechanisms of action and the effectiveness of back belts in the prevention of occupational low back disorders remains uncertain, generating controversy about its use. Therefore, the purpose of this study was to do a critical literature review about back belts use in the prevention of occupational low back pain in the occupational setting. In order to do that, a bibliographic research in the following data basis: Medline, SciELO and LILACS was conducted. There is evidence that back belts use reduces spine range of motion, however the studies about intraabdominal pressure and muscle activity presented contradictory results, as the epidemiologic studies, not allowing the recommendation of its use in the prevention of occupational low back pain. Based on this literature review it is suggested therefore, the implementation of new studies, to confirm or not the effectiveness of back belts as personal protective equipment, in the prevention of occupational low back disorders.

Key-words: Ergonomics, occupational health, occupational risks, back pain, personal protective equipments.

\section{INTRODUÇÃO}

No ambiente ocupacional as disfunções da coluna lombar são as mais comuns e onerosas dentre os distúrbios musculoesqueléticos. Apresentam participação significativa nas causas de absenteísmo ao trabalho, são responsáveis por 15 a $20 \%$ de todas as notificações de doenças e, aproximadamente, $25 \%$ dos Revista Produção Online. Florianópolis, SC, v.12, n. 1, p. 23- 45, jan./mar. 2012. 
casos de invalidez prematura, gerando prejuízos financeiros, perda da qualidade, perda de competitividade e queda na produção das organizações (MARRAS, 2000; IGUTI; HOEHNE, 2004; MATOS et al., 2008).

De acordo com Van Poppel et al. (1998) e Matos et al. (2008) apesar da etiologia da dor lombar ser reconhecidamente multifatorial, as condições de trabalho frequentemente são apontadas como tendo um papel importante na sua gênese. Os fatores de risco em potencial para a dor lombar de origem ocupacional podem ser divididos em três categorias: individuais, biomecânicos e psicossociais.

Segundo a Força-Tarefa Canadense para Cuidados de Saúde Preventiva (CANADIAN TASK FORCE ON PREVENTIVE HEALTH CARE, 2003) o principal fator de risco individual é a história prévia de dor lombar, sendo que idade, obesidade e gênero apresentam uma menor associação. Já entre os fatores biomecânicos, as associações mais consistentes são com exposição ao levantamento e/ou transporte de cargas pesadas, vibração de corpo inteiro, flexão e rotação do tronco frequentes e posturas de trabalho estáticas (VAN POPPEL et al., 1998; MARRAS, 2000; CANADIAN TASK FORCE ON PREVENTIVE HEALTH CARE, 2003; MATOS et al., 2008). Quanto aos fatores psicossociais, existe um corpo de evidências empíricas crescente ligando estes com um maior risco de dor lombar de origem ocupacional, mas a força desta associação ainda é modesta (MARRAS, 2000; WADDELL E BURTON, 2001; CANADIAN TASK FORCE ON PREVENTIVE HEALTH CARE, 2003; FERNANDES et al., 2009).

Desta forma, Wassel et al. (2000) e Van Poppel, Hooftman e Koes (2004) relatam que em resposta aos custos humanos e econômicos dos problemas lombares, os empregadores vêm adotando programas preventivos. De acordo com van Poppel et al. (1998), van Poppel, Hooftman e Koes (2004) e Ammendolia, Kerr e Bombardier (2005) à parte das melhorias ergonômicas realizadas nos postos de trabalhos, uma das estratégias de prevenção mais comumente adotadas no cenário industrial é a utilização de cintos lombares por parte dos colaboradores.

Os cintos lombares, também conhecidos como suportes lombares, cintos abdominais ou ainda cintos lombo-sacrais, nas últimas décadas têm se tornado populares no ambiente ocupacional como forma de prevenção de distúrbios lombares. Teoricamente estes atuariam através de três mecanismos de ação: aumento da pressão intra-abdominal, redução da força muscular e diminuição da 
amplitude de movimento da coluna (BARRON; FEUERSTEIN, 1994; VAN POPPEL et al., 2000).

Entretanto, Minor (1996), Marras, Jorgensen e Davis (2000), Roelofs et al. (2007) e van Duijvenbode et al. (2008) afirmam que embora largamente utilizados, a eficácia dos cintos lombares na prevenção de distúrbios lombares de natureza ocupacional permanece incerta. Assim como os mecanismos de ação ou, ainda seus potencias efeitos adversos (lesões dermatológicas, distúrbios gastrointestinais, diminuição de tônus muscular, aumento da pressão arterial e do ritmo cardíaco), os quais segundo McGill (1993), Minor (1996), Calmels e Fayolle-Minon (1996) e Dillingham (1998) não foram ainda completamente comprovados, suscitando controvérsias quanto à sua utilização.

Diante deste contexto, órgãos de prestígio internacional como o National Institute for Occupational Safety and Health (NIOSH, 1994), o Canadian Center for Occupational Health and Safety (CCOHS, 2005), a European Agency for Safety and Health at Work (EU-OSHA, 2008) e a WorkSafe Victoria (2009), responsáveis pela aplicação de leis sobre saúde e segurança no trabalho nos Estados Unidos, Canadá, Europa e Austrália, respectivamente, não recomendam o uso dos cintos lombares como equipamento de proteção individual no ambiente ocupacional.

Perante o que foi exposto anteriormente, formulou-se à seguinte questão norteadora do estudo: quais são as evidências científicas quanto aos mecanismos de ação e a eficácia dos cintos lombares na prevenção dos distúrbios da coluna lombar no ambiente ocupacional?

Deste modo, a presente pesquisa teve como objetivos, investigar através de uma revisão bibliográfica: a) os mecanismos de ação biomecânicos e fisiológicos dos cintos lombares; assim como b) a eficácia destes na prevenção de distúrbios lombares no contexto ocupacional.

A realização deste estudo foi baseada na pressuposição de que a síntese de evidências referentes à utilização dos cintos lombares no ambiente ocupacional é capaz de embasar de forma científica as decisões quanto à escolha dos métodos de prevenção primária da dor lombar de origem ocupacional e, por conseguinte, gerar resultados de maior efetividade. 


\section{MÉTODO}

O presente estudo caracteriza-se como uma revisão crítica da literatura. De acordo com Mancini e Sampaio (2006) esta forma de revisão é caracterizada pela análise e síntese da informação disponibilizada pelos estudos relevantes sobre um determinado tema, de forma a sintetizar o corpo de conhecimento existente e levar a concluir sobre o assunto de interesse.

A pesquisa foi realizada nas bases de dados Medline, SciELO e LILACS, fazendo uso das seguintes palavras-chave: cintos lombares, suportes lombares, cintos para levantamento de peso e cintos abdominais; assim como os termos correspondentes em inglês: back belts, lumbar support, abdominal belts, weight-belts e lifting belts, no período compreendido entre 1990 e janeiro de 2010.

Inicialmente os estudos foram selecionados com base nos títulos, sendo excluídos aqueles claramente não relacionados com o tema de revisão proposto. A seguir, foram lidos e analisados os resumos de todos os estudos restantes, sendo selecionados os que demonstraram relação direta com a temática deste estudo.

Após a seleção e análise dos artigos, os resultados foram apresentados e discutidos em separado de acordo com os seguintes grupos: pressão intraabdominal, atividade muscular, cinemática e cinética da coluna vertebral e estudos epidemiológicos. Nos três primeiro grupos foram alocados os estudos onde os prováveis mecanismos de ação dos cintos lombares foram testados através de modelos biomecânicos e/ou fisiológicos. Já no último grupo estudos que utilizaram métodos epidemiológicos para determinar a eficácia dos cintos lombares na prevenção de distúrbios lombares no ambiente ocupacional foram analisados.

\section{RESULTADOS E DISCUSSÃO}

A utilização das palavras-chave em português não obteve resultados, já a utilização das mesmas na língua inglesa retornou 121 artigos. Destes 34 apresentaram relação direta com a temática deste estudo, sendo que em 28 os prováveis mecanismos de ação dos cintos lombares foram testados através de modelos biomecânicos e/ou fisiológicos e nos outros 6 foram utilizados métodos epidemiológicos.

Revista Produção Online. Florianópolis, SC, v.12, n. 1, p. 23- 45, jan./mar. 2012. 


\subsection{Pressão Intra-Abdominal}

Quando um objeto é levantado com a coluna vertebral flexionada, um momento flexor causado pela carga externa e o peso da parte superior do tronco é gerado sobre a coluna, este sofre oposição de um momento extensor gerado pelos músculos paravertebrais e do quadril. Hipoteticamente a pressão intra-abdominal (PIA) auxilia no desenvolvimento deste momento extensor, ao produzir uma força anterior à coluna vertebral. Desta maneira a realização de força pelos músculos paravertebrais seria reduzida, o que por sua vez diminuiria as forças compressivas que atuam sobre a coluna vertebral e os discos intervertebrais (MARRAS, JOYNT; KING, 1985; PERKINS; BLOSWICK, 1995).

Apesar do real papel da PIA na redução das forças compressivas que atuam sobre a coluna lombar ser inconclusivo, foi decidido analisar esta variável neste estudo, uma vez que o mesmo é citado por diversos artigos como um possível mecanismo de ação dos cintos lombares.

Cinco estudos avaliaram influência dos cintos lombares sobre a PIA: Lander, Simonton e Giaccobe (1990), McGill et al. (1990), Lander et al. (1992), Woodhouse et al. (1995), Myamoto et al. (1999) (Quadro 1). Os resultados destes estudos foram contraditórios: três apresentaram aumento estatisticamente significativo da PIA quando do uso do cinto lombar enquanto dois não encontraram diferença estatisticamente significativa entre os valores da PIA com o uso do cinto lombar.

Um estudo analisou ainda a pressão extra-abdominal (PEA), Udo e Yoshinaga (1997), observando aumento estatisticamente significativo da mesma, contudo a relação entre a PEA, a pressão dos músculos abdominais e a PIA não foi completamente esclarecida.

\subsection{Atividade Muscular}

O suposto aumento da PIA, imposto pelo uso dos cintos lombares, hipoteticamente, seria capaz de reduzir a força muscular realizada durante o levantamento de materiais, a fadiga muscular resultante desta e, portanto a pressão intra-discal (MCGILL, 1993; MINOR, 1996; VAN POPPEL, et al., 2000). 
Dezesseis estudos analisaram a relação entre o uso do cinto lombar e atividade muscular: Lander, Simonton e Giaccobe (1990), McGill et al. (1990), Lander et al. (1992), Ciriello e Snook (1995), Reyna et al. (1995), Magnusson, Pope e Hansson (1996), Granata, Marras e Davis (1997), Majkowski et al. (1998), Lavender et al. (1998), Cholewicki et al. (1999), Myamoto et al. (1999), Thomas et al. (1999), Lee e Chen (1999), Lavender et al. (2000), Warren et al. (2001), Lee e Kang (2002) (Quadro 2). Destes, quinze fizeram uso da eletromiografia (EMG), sendo que na maioria os pesquisadores avaliaram a EMG dos músculos eretor espinhal e oblíquo abdominal, obtendo resultados conflitantes: cinco apresentaram redução do sinal eletromiográfico, enquanto sete não apresentaram alterações. Já um estudo apresentou aumento estatisticamente significativo da EMG durante a liberação simétrica de uma carga, já durante a liberação de maneira assimétrica a EMG apresentou redução estatisticamente significativa, enquanto que em outro estudo a liberação de forma assimétrica aumentou de forma estatisticamente significativa a EMG. Finalmente, um dos estudos avaliou a EMG do músculo oblíquo abdominal de forma isolada, encontrando redução estatística significativa da EMG dos sujeitos do sexo feminino e resultado inverso para os sujeitos do sexo masculino.

Além da EMG, determinados estudos analisaram outras variáveis relacionadas à atividade muscular como a intensidade percebida, a resistência isocinética, o peso máximo aceitável, a força lombar extensora, a força isométrica e o pico máximo de força, sendo que nenhuma destas variáveis foi afetada pelo uso dos cintos lombares. 
Quadro 1 - Síntese dos estudos sobre a relação entre o uso dos cintos lombares e a PIA

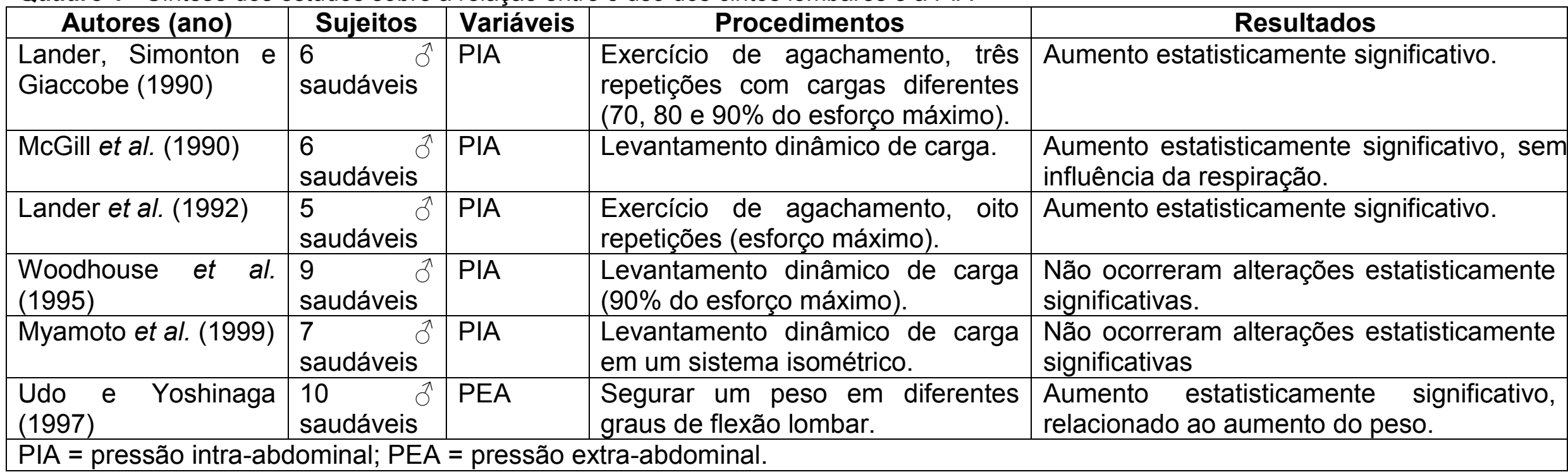




\subsection{Cinemática e Cinética da Coluna Vertebral}

A diminuição da amplitude dos movimentos (ADM) realizados pela coluna vertebral devido ao uso do cinto lombar está relacionada à obstrução física dos movimentos (MINOR, 1996; BARRON; FEUERSTEIN, 1994; VAN POPPEL et al., 2000).

Doze estudos analisaram a influência do uso do cinto lombar sobre variáveis cinemáticas e/ou cinéticas da coluna vertebral: Jonai et al. (1997), Granata, Marras e Davis (1997), Sparto et al. (1998), Thoumie et al. (1998), McGorry e Hsiang (1999), Lavender et al. (2000), Lee e Chen (2000), Marras, Jorgensen e Davis (2000), Willey (2000), Giorcelli et al. (2001), Knigma et al. (2006) e Katsuhira et al. (2008) (Quadro 3). A ADM foi verificada em nove estudos, destes, oito apresentaram redução estatisticamente significativa da ADM da coluna em pelo menos um plano de movimento, e somente um estudo não encontrou alterações da ADM. Deve-se registrar ainda, que quatro dos estudos supracitados encontraram alterações da ADM em articulações adjacentes (pelve, quadril e joelho). 
Quadro 2 - Síntese dos estudos sobre a relação entre o uso dos cintos lombares e atividade muscular

\begin{tabular}{|c|c|c|c|c|}
\hline Autores (ano) & Sujeitos & Variáveis & Procedimentos & Resultados \\
\hline $\begin{array}{l}\text { Lander, Simonton e } \\
\text { Giaccobe (1990) }\end{array}$ & $\begin{array}{l}6 \\
\text { saudáveis }\end{array}$ & EMG & $\begin{array}{l}\text { Exercício de agachamento, três } \\
\text { repetições com cargas diferentes } \\
(70,80 \text { e } 90 \% \text { do esforço máximo). }\end{array}$ & $\begin{array}{l}\text { Redução estatisticamente significativa da } \\
\text { EMG do MEE e MOE. }\end{array}$ \\
\hline Lander et al. (1992) & $\begin{array}{l}5 \\
\text { saudáveis }\end{array}$ & EMG & $\begin{array}{l}\text { Exercício de agachamento, oito } \\
\text { repetições (esforço máximo). }\end{array}$ & $\begin{array}{l}\text { A EMG do MEE e MOE não sofreu } \\
\text { alterações; } \\
\text { Aumento estatisticamente significativo da } \\
\text { EMG do MVL e MBP. }\end{array}$ \\
\hline $\begin{array}{lll}\text { Ciriello } & \text { e } & \text { Snook } \\
(1995) & & \end{array}$ & $\begin{array}{l}13 \quad \hat{0} \\
\text { saudáveis }\end{array}$ & $\begin{array}{l}\text { EMG } \\
\text { IP } \\
\text { RI }\end{array}$ & $\begin{array}{l}\text { Levantamento dinâmico de carga; } \\
\text { Extensão isocinética. }\end{array}$ & $\begin{array}{llll}\text { Nenhuma } & \text { variável } & \text { observada } & \text { sofreu } \\
\text { alterações. } & & \end{array}$ \\
\hline Reyna et al. (1995) & $\begin{array}{l}13 \text { + } 9 \sigma^{1} \\
\text { saudáveis }\end{array}$ & $\begin{array}{l}\text { FLE } \\
\text { PMA }\end{array}$ & $\begin{array}{l}\text { Extensão lombar; } \\
\text { Levantamento dinâmico de carga. }\end{array}$ & $\begin{array}{llll}\text { Nenhuma } & \text { variável } & \text { observada } & \text { sofreu } \\
\text { alterações. } & & & \end{array}$ \\
\hline $\begin{array}{l}\text { Magnusson, Pope e } \\
\text { Hansson (1996) }\end{array}$ & $\begin{array}{l}7 \text { †, } 5 \text { o } \\
\text { saudáveis }\end{array}$ & EMG & Levantamento dinâmico de carga. & $\begin{array}{l}\text { Redução estatisticamente significativa da } \\
\text { EMG dos músculos dorsais. }\end{array}$ \\
\hline $\begin{array}{l}\text { Majkowski et al. } \\
(1998)\end{array}$ & $\begin{array}{l}11+, 13 \AA \\
\text { saudáveis }\end{array}$ & $\begin{array}{l}\text { EMG } \\
\text { FI }\end{array}$ & $\begin{array}{l}\text { Levantamento isométrico máximo; } \\
\text { Levantamento dinâmico de carga. }\end{array}$ & $\begin{array}{lll}\text { Nenhuma } & \text { variável } & \text { observada } \\
\text { alterações. } & & \\
\end{array}$ \\
\hline Lavender et al. (1998) & $\begin{array}{l}2 \text { ㅇ, } 10 \text { त } \\
\text { saudáveis }\end{array}$ & $\begin{array}{l}\text { EMG } \\
\text { PMF }\end{array}$ & $\begin{array}{l}\text { Simulação do movimento de puxar } \\
\text { de forma isométrica, em superfícies } \\
\text { estáveis e escorregadias. }\end{array}$ & 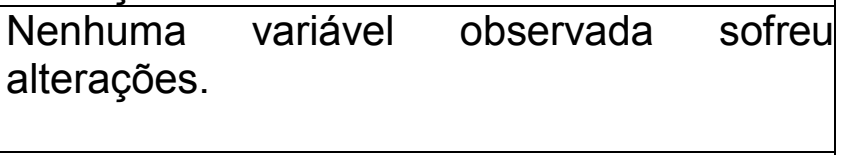 \\
\hline $\begin{array}{l}\text { Cholewicki } \\
\text { (1999) }\end{array}$ & $\begin{array}{l}10 \text { (sexo?) } \\
\text { saudáveis }\end{array}$ & EMG & Liberação súbita de carga. & $\begin{array}{l}\text { Redução estatisticamente significativa da } \\
\text { EMG do MEE. }\end{array}$ \\
\hline
\end{tabular}


Quadro 2- Síntese dos estudos sobre a relação entre o uso dos cintos lombares e atividade muscular

\begin{tabular}{|c|c|c|c|c|}
\hline Autores (ano) & Sujeitos & Variáveis & Procedimentos & Resultados \\
\hline Myamoto et al. (1999) & 7 saudáveis & EMG & $\begin{array}{l}\text { Levantamento dinâmico de carga } \\
\text { em um sistema isométrico. }\end{array}$ & $\begin{array}{l}\text { A EMG do MEE e MOE não sofreu } \\
\text { alterações; } \\
\text { Aumento estatisticamente significativo da } \\
\text { EMG do MRA. }\end{array}$ \\
\hline Thomas et al. (1999) & $\begin{array}{l}10+10 \AA \\
\text { saudáveis }\end{array}$ & EMG & $\begin{array}{l}\text { Liberação súbita de carga simétrica } \\
\text { e assimétrica. }\end{array}$ & $\begin{array}{l}\text { Aumento estatisticamente significativo da } \\
\text { EMG do MEE, na liberação simétrica; } \\
\text { Redução estatisticamente significativa da } \\
\text { EMG do MEE, na liberação assimétrica. }\end{array}$ \\
\hline Lavender et al. (2000) & $\begin{array}{l}8 \text { ㅇ, } 10 \text { đ } \\
\text { saudáveis }\end{array}$ & EMG & $\begin{array}{l}\text { Liberação súbita de carga simétrica } \\
\text { e assimétrica. }\end{array}$ & $\begin{array}{l}\text { Aumento estatisticamente significativo da } \\
\text { EMG do MEE, na liberação assimétrica } \\
\text { apenas; } \\
\text { Redução estatisticamente significativa da } \\
\text { EMG do MOE e MRA. }\end{array}$ \\
\hline Lee e Kang (2002) & $\begin{array}{l}11 \text { o } \\
\text { saudáveis }\end{array}$ & EMG & Levantamento dinâmico de carga. & $\begin{array}{l}\text { Redução estatisticamente significativa da } \\
\text { EMG do MEE; } \\
\text { Aumento estatisticamente significativo da } \\
\text { EMG do MOA e MRA. }\end{array}$ \\
\hline
\end{tabular}


Quatro estudos avaliaram ainda a velocidade angular máxima (VAM), sendo que todos reportaram redução estatisticamente significativa desta variável em pelo menos um plano de movimento.

Finalmente, cinco estudos avaliaram a relação da utilização do cinto lombar e os momentos impostos à coluna, sendo que dois encontraram aumento estatisticamente significativo do momento extensor, um encontrou redução do momento extensor e lateral, outro encontrou redução do momento flexor e, por último, foi encontrado ainda redução estatisticamente significativa do momento de rotação em um dos estudos.

\subsection{Estudos Epidemiológicos}

Para Minor (1996) o resultado de qualquer intervenção preventiva é uma das medidas mais importante na avaliação de sua eficiência, sendo assim Reddell et al. (1992), Mitchell et al. (1994), Kraus et al. (1996), Van Poppel et al. (1998), Wassel et al. (2000) e Roelofs et al. (2007) realizaram estudos epidemiológicos a fim de coletar evidências que justifiquem ou não o uso destes equipamentos.

Reddell et al. (1992) avaliaram a eficácia de um cinto lombar na redução da taxa de incidência de lesões da coluna lombar em um período de oito meses. $\mathrm{O}$ estudo foi realizado em uma companhia aérea com 642 carregadores de bagagem. Estes foram divididos de forma aleatória em 4 grupos: no primeiro fez-se uso somente do cinto lombar, no segundo os trabalhadores receberam treino quanto ao levantamento de cargas durante uma hora, no terceiro foi associado o uso do cinto lombar ao treino de levantamento, e um grupo controle onde não foi realizada nenhuma intervenção. A concordância com o uso dos cintos lombares foi um fator relevante; no primeiro grupo $61 \%$ dos indivíduos descontinuaram o uso do cinto lombar antes da conclusão do estudo, enquanto que no terceiro grupo esse valor foi de 58\%. Os resultados indicaram não haver diferenças estatisticamente significativas entre os quatro grupos quanto à taxa de incidência de lesões, dias de trabalho perdidos devido a lesões, dias de trabalho perdidos e taxa de uso do seguro social. Entretanto, foi observado um aumento estatisticamente significativo de dias de trabalho perdidos devido a lesões nos participantes que fizeram uso dos cintos por um período e, então descontinuaram o seu uso. O que, segundo os autores pode 
Quadro 3 - Síntese dos estudos sobre a relação entre o uso dos cintos lombares e a cinemática da coluna vertebral

(Continua)

\begin{tabular}{|c|c|c|c|c|}
\hline Autores (ano) & Sujeitos & Variáveis & Procedimentos & Resultados \\
\hline Jonai et al. (1997) & $\begin{array}{l}12 \text { (sexo?) } \\
\text { saudáveis }\end{array}$ & $\begin{array}{l}\text { ADM } \\
\text { VAM }\end{array}$ & $\begin{array}{l}\text { Análise de um dia de trabalho } \\
\text { normal, de tarefas que envolviam } \\
\text { levantamento dinâmico de carga. }\end{array}$ & $\begin{array}{l}\text { Não ocorreram alterações estatisticamente } \\
\text { significativas da ADM; } \\
\text { Redução estatisticamente significativa da } \\
\text { VAM de flexão do tronco. }\end{array}$ \\
\hline $\begin{array}{l}\text { Granata, Marras e } \\
\text { Davis (1997) }\end{array}$ & $\begin{array}{l}15 \text { saudáveis } \\
\text { sa }\end{array}$ & $\begin{array}{l}\text { ADM } \\
\text { VAM } \\
\text { Momento }\end{array}$ & $\begin{array}{l}\text { Levantamento dinâmico de carga, } \\
\text { simétrico e assimétrico, com três } \\
\text { tipos de cintos diferentes. }\end{array}$ & $\begin{array}{l}\text { Redução estatisticamente significativa da } \\
\text { ADM da coluna nos três planos (somente } \\
\text { com cinto elástico); } \\
\text { Aumento estatisticamente significativo da } \\
\text { ADM da pelve nos planos sagital e } \\
\text { transversal; } \\
\text { Redução estatisticamente significativa da } \\
\text { VAM nos três planos; } \\
\text { Aumento estatisticamente significativo do } \\
\text { momento extensor (somente com cinto } \\
\text { ortótico). }\end{array}$ \\
\hline $\begin{array}{l}\text { McGorry e Hsiang } \\
\text { (1999) }\end{array}$ & $\begin{array}{l}6 \\
\text { saudáveis }\end{array}$ & ADM. & $\begin{array}{l}\text { Levantamento dinâmico de carga, } \\
\text { com dois tipos de cintos diferentes. }\end{array}$ & $\begin{array}{l}\text { Redução estatisticamente significativa da } \\
\text { ADM da coluna no plano sagital; } \\
\text { Não ocorreram alterações estatisticamente } \\
\text { significativas da ADM da pelve. }\end{array}$ \\
\hline
\end{tabular}


Quadro 3 - Síntese dos estudos sobre a relação entre o uso dos cintos lombares e a cinemática da coluna vertebral

(Continuação)

\begin{tabular}{|c|c|c|c|c|}
\hline Autores (ano) & Sujeitos & Variáveis & Procedimentos & Resultados \\
\hline $\begin{array}{l}\text { Lavender et al. } \\
(2000)\end{array}$ & $\begin{array}{l}8 \text { + }, 10 \text { त } \\
\text { saudáveis }\end{array}$ & $\begin{array}{l}\text { ADM } \\
\text { Momento }\end{array}$ & $\begin{array}{l}\text { Liberação súbita de carga } \\
\text { simétrica e assimétrica. }\end{array}$ & $\begin{array}{l}\text { Redução estatisticamente significativa da ADM da } \\
\text { coluna no plano sagital e frontal; } \\
\text { Não ocorreram alterações estatisticamente } \\
\text { significativas da ADM da pelve; } \\
\text { Redução estatisticamente significativa do momento } \\
\text { de flexão da coluna. }\end{array}$ \\
\hline Lee e Chen (2000) & $\begin{array}{l}18 \quad 0 \\
\text { saudáveis }\end{array}$ & $\begin{array}{l}\text { Ângulo } \\
\text { L1/S1 }\end{array}$ & $\begin{array}{l}\text { Manutenção das posturas } \\
\text { em pé, sentado e sentado } \\
\text { com flexão de tronco. }\end{array}$ & $\begin{array}{l}\text { Aumento estatisticamente significativo do ângulo } \\
\text { L1/S1 em pé e sentado com flexão de tronco (cinto } \\
\text { lombar e pélvico); } \\
\text { Redução estatisticamente significativa do ângulo } \\
\text { L1/S1 quando sentado (somente com cinto } \\
\text { pélvico). }\end{array}$ \\
\hline $\begin{array}{l}\text { Marras, Jorgensen e } \\
\text { Davis (2000) }\end{array}$ & $\begin{array}{l}20 \quad 0^{\pi} \\
\text { saudáveis }\end{array}$ & $\begin{array}{l}\text { ADM } \\
\text { Momento }\end{array}$ & $\begin{array}{l}\text { Levantamento dinâmico de } \\
\text { carga, simétrico } \\
\text { assimétrico. }\end{array}$ & $\begin{array}{l}\text { Redução estatisticamente significativa da ADM } \\
\text { sagital e transversal da coluna; } \\
\text { Aumento da ADM do quadril associado à redução } \\
\text { da ADM do joelho, ambos estatisticamente } \\
\text { significativos e ambos no plano sagital; } \\
\text { Redução estatisticamente significativa do momento } \\
\text { de rotação da coluna. }\end{array}$ \\
\hline Giorcelli et al. (2001) & 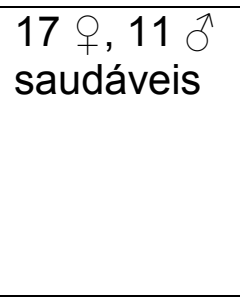 & $\begin{array}{l}\text { ADM } \\
\text { VAM }\end{array}$ & $\begin{array}{l}\text { Levantamento dinâmico de } \\
\text { carga, assimétrico. }\end{array}$ & $\begin{array}{l}\text { Redução estatisticamente significativa da ADM da } \\
\text { coluna nos três planos; } \\
\text { Aumento estatisticamente significativo da ADM do } \\
\text { quadril e joelho no plano sagital; } \\
\text { Redução estatisticamente significativa da VAM } \\
\text { nos planos sagital e frontal. }\end{array}$ \\
\hline
\end{tabular}


Quadro 3 - Síntese dos estudos sobre a relação entre o uso dos cintos lombares e a cinemática da coluna vertebral

\begin{tabular}{|c|c|c|c|c|}
\hline Autores (ano) & Sujeitos & Variáveis & Procedimentos & Resultados \\
\hline Kingma et al. (2006) & $\begin{array}{l}9 \\
\text { saudáveis }\end{array}$ & Momento & $\begin{array}{l}\text { Levantamento dinâmico de carga, } \\
\text { durante a inspiração e expiração. }\end{array}$ & $\begin{array}{l}\text { Aumento estatisticamente significativo do } \\
\text { momento extensor apenas quando } \\
\text { associado à inspiração. }\end{array}$ \\
\hline $\begin{array}{l}\text { Katushira et al. } \\
(2008)\end{array}$ & $\begin{array}{l}10 \\
\text { saudáveis } \\
\text { (?) }\end{array}$ & Momento & $\begin{array}{l}\text { Simulação de transferência de } \\
\text { pacientes. }\end{array}$ & $\begin{array}{l}\text { Redução estatisticamente significativa dos } \\
\text { momentos extensor e lateral. }\end{array}$ \\
\hline
\end{tabular}


indicar que o uso dos cintos é capaz de aumentar o risco de lesões no caso de descontinuidade do uso destes.

Mitchell et al. (1994) realizaram uma investigação retrospectiva da eficácia dos cintos lombares em 1.316 indivíduos que desempenhavam tarefas de transporte e levantamento de cargas em uma base da força aérea dos Estados Unidos, durante um período de 7 anos. Dos 1.316 participantes, 38 (2,9\%) apresentaram distúrbios lombares e após avaliação médica foram obrigados a usar cintos lombares, já 172 $(13,1 \%)$ optaram livremente pelo uso destes, enquanto que os $1.106(84 \%)$ indivíduos restantes não fizeram uso dos cintos. A comparação dos dados obtidos demonstrou que os trabalhadores que utilizaram os cintos apresentaram uma taxa menor de dias de trabalho perdidos, contudo não houve diferença estatisticamente significativa na taxa de lesões da coluna lombar. A análise de custo de tratamento dos trabalhadores que sofreram lesões enquanto usavam o cinto lombar demonstrou ser maior que o custo de tratamento dos que sofreram lesões sem o uso do cinto.

Os dados obtidos por Mitchell et al. (1994) devem ser observados com cautela, uma vez que o estudo apresenta certas limitações. Em primeiro lugar, durante o período investigado pelos autores foram utilizados dois tipos diferentes de cinto lombar, o que pode ter influenciado o resultado. Segundo, alguns dos indivíduos que utilizaram o cinto lombar já haviam apresentado episódios de dor lombar, o que pode ter contribuído no aumento dos custos do tratamento destes indivíduos.

Já Kraus et al. (1996), a fim de determinar o efeito do uso dos cintos lombares, analisaram as horas de trabalho/exposição, o uso do cinto lombar, a intensidade de levantamento de materiais e os registros de episódios de dor lombar no período compreendido entre 1989 a 1994 de quase 36.000 funcionários de uma rede varejista de venda de materiais para casa e construção. De acordo com os autores a concordância com o uso mandatório dos cintos foi de $98 \%$. Com base nos dados analisados os autores calcularam a densidade de incidência de episódios de dor lombar aguda, e observaram que antes da implementação de uma política de uso obrigatório dos cintos lombares em 1990 os colaboradores apresentavam 30,6 episódios de dor lombar aguda para cada milhão de horas trabalhadas. E, após a implementação desta política este número caiu para 20,2 episódios para cada milhão de horas trabalhadas, uma redução de 34\%. 
Por outro lado, Van Poppel et al. (1998) pesquisaram a eficácia dos cintos lombares e da educação na prevenção da dor lombar em trabalhadores do departamento de cargas de uma empresa aérea. Um total de 312 trabalhadores foi dividido em 4 grupos de forma randômica. O grupo 1 recebeu instruções de levantamento de materiais e um cinto lombar, o grupo 2 recebeu somente as instruções, o grupo 3 recebeu somente o cinto lombar e o grupo 4 não sofreu nenhuma forma de intervenção (grupo controle). As instruções quanto às técnicas de levantamento tiveram uma duração total de 5 horas, quanto ao cinto lombar este foi recomendado que fosse usado durante as horas de trabalho por 6 meses. A concordância com o uso dos cintos lombares neste estudo foi de $43 \%$. Os resultados demonstraram não haver diferença estatisticamente significativa tanto na incidência de dor lombar quanto nos dias de trabalho perdidos devido à dor lombar, quando comparados os grupos onde houve alguma forma de intervenção com o grupo controle. Entretanto, uma análise de subgrupos revelou que o uso de cintos lombares aumentou de forma estatisticamente significativa os dias de trabalho perdidos devido à dor lombar nos participantes que nunca haviam apresentado dor lombar antes do início da pesquisa. Enquanto que em indivíduos que apresentavam dor lombar no começo da intervenção, o uso destes reduziu de forma estatisticamente significativa o número de dias por mês com dor lombar. Contudo, resultados de análise de subgrupos não devem ser aceitos até que os mesmos sejam confirmados pelos resultados de outros estudos segundo os autores.

Wassel et al. (2000) avaliaram a eficácia do uso de cintos lombares na redução de pedidos de indenização por lesões da coluna e dor lombar em trabalhadores de 160 lojas de uma empresa do setor de varejo. Das 160 lojas estudadas, 89 exigiam o uso do cinto lombar e nas 71 restantes o uso deste era voluntário. Foram incluídos no estudo 13.873 funcionários identificados pela gerência das lojas como envolvidos em tarefas de manuseio e transporte de cargas, os quais forneceram 9.377 entrevistas iniciais e 6.311 entrevistas de acompanhamento. A concordância com o uso diário do cinto lombar nas lojas onde o uso deste era obrigatório foi de $58 \%$, enquanto que nas lojas onde o uso era voluntário este valor foi de 33\%. Quanto aos pedidos de indenização por lesões da coluna e relatos de dor lombar não foram encontradas diferenças estatisticamente significativas quando comparados os dados dos funcionários que relataram usar o 
cinto todos os dias com os dados dos funcionários que relataram nunca usar o cinto ou ainda, quando comparados os dados dos funcionários que relataram usar o cinto uma ou duas vezes na semana com os dados dos funcionários que relataram nunca usar o cinto.

Roelofs et al. (2007) investigaram a eficácia dos cintos lombares na prevenção secundária da dor lombar em 360 atendentes domiciliares com história prévia de dor lombar. Os participantes foram separados em dois grupos de forma aleatória. O grupo controle recebeu instruções sobre métodos de trabalho saudáveis, e o grupo experimental fez uso de um cinto lombar. Os participantes do grupo experimental podiam escolher entre 4 modelos diferentes de cinto lombar, sendo instruídos a utilizá-los nos dias em que os mesmos apresentassem ou acreditassem que poderiam desenvolver dor lombar. Os resultados, após 12 meses de pesquisa, demonstraram que os participantes do grupo experimental apresentaram em média 52,7 dias a menos de dor lombar por ano $(p<0,001)$, porém não foi encontrada diferença estatisticamente significativa entre os grupos quanto aos dias de trabalho perdido devido à doenças. Foi observada ainda uma diferença pequena, mas estatisticamente significativa na intensidade da dor e na capacidade funcional a favor dos indivíduos do grupo experimental.

\section{CONSIDERAÇÕES}

Em termos gerais, os resultados desta pesquisa indicaram que os estudos que investigaram a relação entre as amplitudes de movimento da coluna e o uso dos cintos lombares demonstram um efeito potencial destes na redução da ADM da coluna. Confirmando, assim, que o uso dos cintos lombares pode ter alguma importância na prevenção dos distúrbios lombares de origem ocupacional. Entretanto, as variações na ADM da pelve, do quadril e do joelho, encontradas em diversos estudos, evidenciam a necessidade da realização de estudos que averiguem o risco de lesões em outras articulações.

Já os estudos referentes ao aumento da PIA e da diminuição do esforço e da fadiga muscular apresentam resultados contraditórios, que impossibilitam a elaboração de uma afirmação conclusiva sobre estes possíveis mecanismos de ação dos cintos lombares. A falta de congruência dos resultados destes estudos pode 
estar relacionada aos diferentes métodos e/ou equipamentos utilizados, e com o número limitado de participantes.

Da mesma forma, os estudos epidemiológicos sobre a eficácia dos cintos lombares, além de limitados, apresentam resultados conflitantes, não permitindo a recomendação do seu uso na prevenção da dor lombar de origem ocupacional.

Não obstante, independentemente do motivo, os mecanismos de ação dos cintos lombares, redução da ADM da coluna vertebral à parte, e sua eficiência na prevenção dos distúrbios lombares de origem ocupacional não foram comprovados de forma definitiva até o presente momento. Assim sendo, atualmente o uso dos cintos lombares não é recomendado, dado o fato de não haver suporte científico sobre sua eficácia e, mais grave, sobre os possíveis efeitos adversos à saúde que o uso destes equipamentos pode ocasionar.

À vista destes fatos, torna-se necessário a realização de novos estudos aplicados, a fim de confirmar ou não a eficácia dos cintos lombares como equipamento de proteção individual, na prevenção dos distúrbios lombares.

\section{REFERÊNCIAS}

AMMENDOLIA, C.; KERR, M.S.; BOMBARDIER, C. Back belt use for occupational low back pain: a systematic review. Journal of Manipulative and Physiological Therapeutics, Lombard, v. 28, n. 2, p. 128-134, fev. 2005.

BARRON, B.A.; FEUERSTEIN, M. Industrial back belts and low back pain: mechanisms and outcomes. Journal of Occupational Rehabilitation, Nova lorque, v. 4 , n. 3, p. 125-139, set. 1994.

CALMELS, P.; FAYOLLE-MINON, I. An update on orthotic devices for the lumbar spine based on a review of the literature. Revue du Rhumatisme, Paris, v. 63, n. 4, p. 285-291, abr. 1996.

CANADIAN TASK FORCE ON PREVENTIVE HEALTH CARE. Use of back belts to prevent occupational low-back pain. Recommendation statement from the Canadian Task Force on Preventive Health Care. Canadian Medical Association Journal, Ottawa, v. 169, n. 3, p. 213-214, ago. 2003.

CCOHS. Back belts. Disponível em

$<$ http://www.ccohs.ca/oshanswers/ergonomics/back blt.html>. Acesso em: 20 mar. 2009.

CHOLEWICKI, J.; JULURU, K.; RADEBOLD, A.; PANJABI, M.M.; MCGILL, S.M. Lumbar spine stability can be augmented with an abdominal belt and/or increased 
intra-abdominal pressure. European Spine Journal, Heidelberg, v. 8, n. 5, p. 388395, 1999.

CIRIELLO, V.M., SNOOK, S.H. The effect of back belts on lumbar muscle fatigue. Spine. Londres, v. 20, n. 11, p. 1271-1278, jun. 1995.

DILLINGHAM, T.R. Lumbar supports for prevention of low back pain in the workplace. JAMA, Chicago, v. 279, n. 22, p. 1826-1828, jun. 1998.

EU-OSHA. Factsheet 78 - Work-related musculoskeletal disorders: prevention report. Disponível em: <http://osha.europa.eu/en/publications/factsheets/78/view>. Acesso em: 20 mar. 2009.

FERNANDES, R.C.P.; CARVALHO, F.M.; ASSUNÇÃO, A.A.; SILVANY NETO, A.M. Interactions between physical and psychosocial demands of work associated to low back pain. Revista de Saúde Pública, São Paulo, v. 43, n. 2, p. 326-334, abr. 2009.

GIORCELLI, R.J.; HUGHES R.E.; WASSELL, J.T.; HSIAO, H. The Effect of Wearing a Back Belt on Spine Kinematics During Asymmetric Lifting of Large and Small Boxes. Spine, Londres, vol. 26, n. 16, p. 1794-1798, ago. 2001.

GRANATA, K.P.; MARRAS, W.S.; DAVIS, K.G. Biomechanical assessment of lifting dynamics, muscle activity and spinal loads while using three different styles of lifting belt. Clinical Biomechanics, Oxford, v. 12, n. 2, p. 107-115, mar. 1997.

IGUTI, A.M., HOEHNE, E.L. Lombalgias e trabalho. Revista Brasileira de Saúde Ocupacional, São Paulo, v. 28, n. 107/108, p. 73-89, jun. 2004.

JONAI, H.; VILLANUEVA, M.B.; SOTOYAMA, M.; HISANGA, N.; SAITO, S. The effect of a back belt on torso motion--survey in an express package delivery company. Industrial Health, Kawasaki, v. 35, n. 2, p. 235-242, abr. 1997.

KATUSHIRA, J.; SASAKI, H.; ASAHARA, S.; IKEGAMI, T.; ISHIHARA, H.; KIKUCHI, T.; HIRAI, Y.; YAMASAKI, Y.; WADA, T.; MARUYAMA, H. Comparison of low back joint moment using a dynamic 3D biomechanical model in different transferring tasks wearing low back belt. Gait Posture, Oxford, v. 28, n. 2, p. 258-264, ago. 2008.

KINGMA, I.; FABER, G.S.; SUWARGANDA, E.K.; BRUIJNEN, T.B.; PETERS, R.J.; VAN DIEËN, J.H. Effect of a stiff lifting belt on spine compression during lifting.

Spine, Londres, v. 31, n. 22, p. E833-E839, out. 2006.

KRAUS, J.F.; BROWN, K.A.; MCARTHUR, D.L.; PEEK-ASA, C.; SAMANIEGO, L.; KRAUS, C. Reduction of Acute Low Back Injuries by Use of Back Supports.

International Journal of Occupational Medicine and Environmental Health, Lodz, v. 2, n. 4, p. 264-273, out. 1996.

LANDER, J.E.; HUNDLEY, J.R.; SIMONTON, R.L. The effectiveness of weight-belts during multiple repetitions of the squat exercise. Medicine and Science in Sports and Exercise, Madison, v. 24, n. 5, p. 603-609, maio 1992. 
LANDER, J.E.; SIMONTON, R.L.; GIACOBBE, J.K. The effectiveness of weight-belts during the squat exercise. Medicine and Science in Sports and Exercise, Madison, v. 22 , n. 1 , p. $117-126$, fev. 1990.

LAVENDER, S.A.; SHAKEEL, K.; ANDERSSON, G.B.; THOMAS, J.S. Effects of lifting belt on spine moments and muscle recruitments after unexpected sudden loading. Spine, Londres, v. 25, n. 12, p. 1569-1578, jun. 2000.

LAVENDER, S.A.; CHEN, S.H.; LI, Y.C.; ANDERSSON, G.B. Trunk muscle use during pulling tasks: effects of a lifting belt and footing conditions. Human Factors, Nova lorque, v. 40, n. 1, p. 159-172, mar. 1998.

LEE, Y.; CHEN, C. Belt effects on lumbar sagittal angles. Clinical Biomechanics, Oxford, v. 15, n. 2, p. 79-82, fev. 2000.

LEE, Y.; CHEN, C. Lumbar vertebral angles and back muscle loading with belts. Industrial Health, Kawasaki, v. 37, n. 4, p. 390-397, 1999.

LEE, Y.; KANG, S. Effect of belt pressure and breath held on trunk electromyography. Spine, Londres, v. 27, n. 3, p. 282-290, fev. 2002.

MAGNUSSON, M.; POPE, M.H.; HANSSON, T. Does a back support have a positive biomechanical effect? Applied Ergonomics, Oxford, v. 27, n. 3, p. 201-205, jun. 1996.

MAJKOWSKI, G.R.; The Effect of Back Belt Use on Isometric Lifting Force and Fatigue of the Lumbar Paraspinal Muscles The Effect of Back Belt Use on Isometric Lifting Force and Fatigue of the Lumbar Paraspinal Muscles. Spine, Londres, v. 23, n. 19, p. 2104-2109, out. 1998.

MANCINI, M.C.; SAMPAIO, R.F. Quando o objeto de estudo é a literatura: estudos de revisão (Editorial). Revista Brasileira de Fisioterapia, São Carlos, v. 10, n. 4, p. 361-361, out./dez. 2006.

MARRAS, W.S. Occupational low back disorder causation and control. Ergonomics, Londres, v. 43, n. 7, p. 880-902, jul. 2000.

MARRAS, W.S.; JORGENSEN, M.J.; DAVIS, K.G. Effect of foot movement and an elastic lumbar back support on spinal loading during free-dynamic symmetric and asymmetric lifting exercises. Ergonomics, Londres, v. 43, n. 5, p. 653-668, maio 2000.

MARRAS, W.S.; JOYNT, R.L.; KING, A.I. The force-velocity and intra-abdominal pressure during lifting activities. Ergonomics, Londres, v. 28, n. 3, p. 603-613, mar. 1985.

MATOS, M.G.; HENNINGTON, E.A., HOEFEL, A.L.; DIAS-DA-COSTA, J.S. Dor lombar em usuários de um plano de saúde: prevalência e fatores associados.

Cadernos de Saúde Pública, Rio de Janeiro, v. 24, n. 9, p. 2115-2122, set. 2008. 
MCGILL, S.M. Abdominal belts in industry: a position paper on their assets, liabilities and use. American Industrial Hygiene Association Journal, Baltimore, v. 54, n. 12, p. 752-754, dez. 1993.

MCGILL, S.M.; NORMAN, R.W.; SHARRATT, M.T. The effect of an abdominal belt on trunk muscle activity and intra-abdominal pressure during squat lifts.

Ergonomics, Londres, v. 33, n. 2, p. 147-160, fev. 1990.

MCGORRY, R.W.; HSIANG, S.M. The effect of industrial back belts and breathing technique on trunk and pelvic coordination during a lifting task. Spine, Londres, v. 24, n. 11, p. 1124-1130, jun. 1999.

MINOR, S.D. Use of back belts in occupational settings. Physical Therapy, Nova Iorque, v. 76, n. 4, p. 403-408, abr. 1996.

MITCHELL, L.V.; LAWLER, F.H.; BOWEN, D.; MOTE, W.; ASUNDI, P.; PURSWELL, J. Effectiveness and cost-effectiveness of employer-issued back belts in areas of high risk for back injury. Journal of Occupational Medicine, Chicago, v. 36, n. 1, p. 90-94, jan. 1994.

MIYAMOTO, K.; IINUMA, N.; MAEDA, M.; WADA, E.; SHIMIZU, K. Effects of abdominal belts on intra-abdominal pressure, intra-muscular pressure in the erector spinae muscles and myoelectrical activities of trunk muscles. Clinical

Biomechanics, Oxford, v. 14, n. 2, p. 79-87, fev. 1999.

$\mathrm{NIOSH}$. Workplace use of back belts: review and recommendations. Washington, DC: US Department of Health and Human Services, Public Health Service, Centers for Disease Control and Prevention, 1994. 33 p.

PERKINS, M.S.; BLOSWICK, D.S. The use of back belts to increase intraabdominal pressure as a means of preventing low back injuries: a survey of the literature.

International Journal of Occupational Medicine and Environmental Health, Lodz, v. 1, n. 4, p. 326-335, out. 1995.

REDDELL, C.R.; CONGLETON, J.J.; DALE HUCHINGSON, R.; MONTGOMERY, J.F. An evaluation of a weightlifting belt and back injury prevention training class for airline baggage handlers. Applied Ergonomics, Oxford, v. 23, n. 5, p. 319-329, out. 1992.

REYNA Jr., J.R.; LEGGETT, S.H.; KENNEY, K.; HOLMES, B.; MOONEY, V. The effect of lumbar belts on isolated lumbar muscle strength and dynamic capacity. Spine, Londres, v. 20, n. 1, p. 68-73, jan. 1995.

ROELOFS, P.D.D.M.; BIERMA-ZEINSTRA, S.M.; VAN POPPEL, M.N.; JELLEMA, P.; WILLEMSEN, S.P.; VAN TULDER, M.W.; VAN MECHELEN, W.; KOES, B.W. Lumbar supports to prevent recurrent low back pain among home care workers. Annals of Internal Medicine, Filadélfia, v. 147, n. 10, p. 685-692, nov. 2007.

SPARTO, P.J.; PARNIANPOUR, M.; REINSEL, T.E.; SIMON, S. The effect of lifting Revista Produção Online. Florianópolis, SC, v.12, n. 1, p. 23- 45, jan./mar. 2012. 
belt use on multijoint motion and load bearing during repetitive and asymmetric lifting. Journal of Spinal Disorders, Nova lorque, v. 11, n. 1, p. 57-64, fev. 1998.

THOMAS, J.S.; LAVENDER, S.A.; CORCOS, D.M.; ANDERSSON, G.B. Effect of lifting belts on trunk muscle activation during a suddenly applied load. Human Factors, Nova lorque, v. 41, n. 4, p. 670-676, dez. 1999.

THOUMIE, P.; DRAPE, J.L.; AYMARD, C.; BEDOISEAU, M. Effects of a lumbar support on spine posture and motion assessed by electrogoniometer and continuous recording. Clinical Biomechanics, Oxford, v. 13, n. 1, p. 18-26, jan. 1998.

UDO, H.; YOSHINAGA, F. Effect of a pelvic belt on abdominal pressure by various weights and bending angles. Industrial Health, Kawasaki, v. 35, n. 2, p. 229-234, abr. 1997.

VAN DUIJVENBODE, I.; JELLEMA, P.; VAN POPPEL, M.N.; VAN TULDER, M.W. Lumbar supports for prevention and treatment of low back pain. Cochrane Database of Systematic Reviews, Oxford, v. 2, 2008. Disponível em:

$<$ http://mrw.interscience.wiley.com/cochrane/clsysrev/articles/CD001823/frame.html> Acesso em: 20 mar. 2009.

VAN POPPEL, M.N.M.; KOES, B.W.; VAN DER PLOEG, T.; SMID, T.; BOUTER, L.M. Lumbar supports and education for the prevention of low back pain in industry: a randomized controlled trial. JAMA, Chicago, v. 279, n. 22, p. 1789-1794, jun. 1998.

VAN POPPEL, M.N.M.; DE LOOZE, M.P.; KOES, B.W.; SMID, T.; BOUTER, L.M. Mechanisms of action of lumbar supports. Spine, Londres, v. 25, n. 16, p. 21032113, ago. 2000.

VAN POPPEL, M.N.M.; HOOFTMAN, W.E.; KOES, B.W. An update of a systematic review of controlled clinical trials on the prevention of back pain at the worplace.

Occupational Medicine, Londres, v. 54, n. 5, 345-352, ago. 2004.

WADDELL, G.; BURTON, A.K. Occupational health guidelines for the management of low back pain at work: evidence review. Occupational Medicine, Chicago, v.. 52, n. 2, p. 124-135, mar. 2001.

WARREN, L.P.; APPLING, S.; OLADEHIN, A.; GRIFFIN, J. Effect of soft lumbar support belt on abdominal oblique muscle activity in nonimpaired adults during squat lifting. Journal of Orthopaedic and Sports Physical Therapy, Washington, v. 31, n. 6, p.316-323, jun. 2001.

WASSEL, J.T.; GARDNER, L.I.; LANDSITTEL, D.P.; JOHNSTON, J.J.; JOHNSTON, J.M. A prospective study of back belts for prevention of back pain and injury. JAMA. Chicago, v. 284, n. 21, p. 2727-2732, dez. 2000.

WILLEY, M.S. The effects of back belts and load on selected lifting kinematics during a simulated patient transfer. Work: a journal of prevention, assessment, and 
rehabilitation, Amsterdã, v. 17, n. 1, p. 31-38, 2001.

WOODHOUSE, M.L.; MCCOY, R.W.; REDONDO, D.R.; SHALL, L.M. Effects of back support on intra-abdominal pressure and lumbar kinetics during heavy lifting. Human Factors, Nova lorque, v. 37, n. 3, p. 582-590, set. 1995.

WORKSAFE VICTORIA. Back belts are not effective in reducing back injuries. Disponível em:

$<$ http://www.worksafe.vic.gov.au/wps/wcm/connect/wsinternet/worksafe/home/forms+ and+publications/guidance+notes/back+belts+are+not+effective+in+reducing+back+i njuries>. Acesso em: 01 set. 2009.

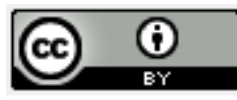

Artigo recebido em 01/06/2010 e aceito para publicação em 23/01/2012. 